\title{
A NOTE ON THE PENETRATION OF A RIGID WEDGE INTO A NONISOTROPIC BRITTLE MATERIAL
}

\author{
R. BenJumea and D. L. SiKarskie \\ The University of Michigan, Ann Arbor, Michigan
}

(Received 30 October 1968)

\begin{abstract}
In the present note an existing wedge penetration theory for isotropic brittle materials is extended to explain some 'bedding plane' effects observed in a series of experiments on Indiana limestone.

The general features of the theory presented by PAUL and SIKARSKIE [Trans. AIME 232, 372-383 (1965)] are kept here and in order to extend the analysis to the anisotropic case Jaeger's modification of the Coulomb-Mohr failure criteria is used.

Two specific cases of bedding plane orientation are considered, namely those in which the bedding planes are parallel and perpendicular to the direction of penetration.

The theory presented gives an insight into the anisotropic effects and predicts, in reasonable agreement with the experimental results, the observed differences in specific energies for the different penetration directions.
\end{abstract}

\section{NOTATION}

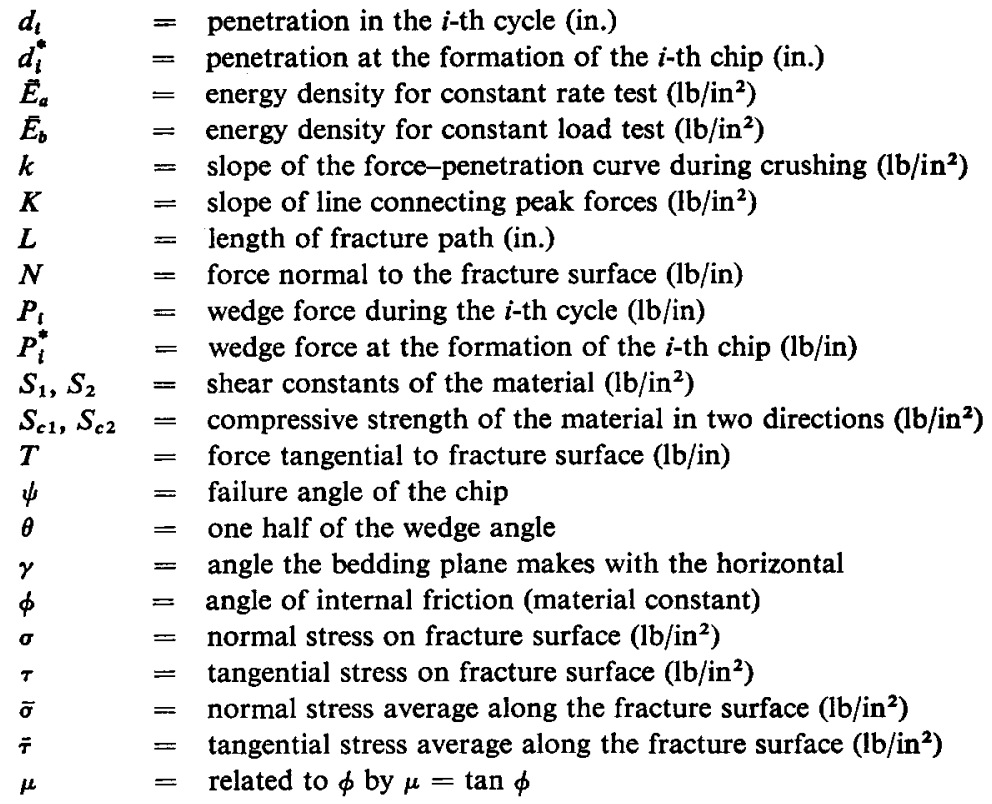

\section{INTRODUCTION}

IN THE PRESENT work an existing wedge penetration theory for brittle materials [1] is extended in an attempt to explain some 'bedding plane' effects observed in a series of experiments on Indiana limestone. Bedding plane effect or rock anisotropy is often neglected 
in Rock Mechanics calculations particularly for rocks such as Indiana limestone where there is no discernible bedding plane and further the change in physical properties, e.g. compressive strength, with direction is relatively small. The present theory (substantiated by experiment) indicates, however, that relatively large differences in the specific energy (energy necessary to remove a unit volume of rock) can exist even for relatively small anisotropy.

The analysis presented herein follows that of [1] and contains the same basic assumptions. The material behavior under consideration is typically brittle, i.e. as the wedge penetrates cycles of crushing followed by the formation of chips are experienced. To extend the analysis to the anisotropic case JAEGER's [2,3] modification of the Coulomb-Mohr failure criterion is used.

Two special cases are considered in this work, namely $\gamma=0^{\circ}, 90^{\circ}$ (see Fig. 1) where $\gamma$ represents the angle the bedding plane makes with the horizontal. Intermediate values of $\gamma$ represent a much more difficult problem since symmetry cannot be invoked. This necessitates some description of the wedge kinematics.

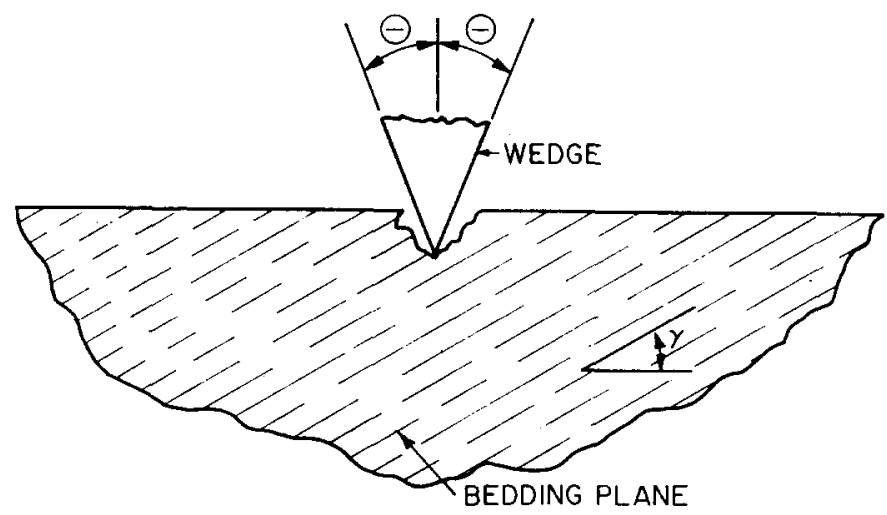

FIG. 1. Bedding plane direction.

In the following section theoretical results are derived. A comparison of the theory with some previously unpublished [4] wedge penetration experiments on Indiana limestone is given in Section 3 followed by a discussion of results in Section 4.

\section{THEORETICAL ANALYSIS}

Figure 2 illustrates the assumed idealized penetration model just at the formation of the $(i+1)$ st chip. Chip failure is assumed planar and inclined to the horizontal at some as yet unknown angle $\psi$. Considering equilibrium just before formation of the chip gives the following for the normal and tangential forces on the failure plane: $\dagger$

$$
\begin{aligned}
& N=\frac{P_{i+1}^{*}}{2} \frac{\sin (\psi+\theta)}{\sin \theta} \\
& T=\frac{P_{i+1}^{*}}{2} \frac{\cos (\psi+\theta)}{\sin \theta}
\end{aligned}
$$

† Note that the wedge is assumed frictionless. This assumption can be removed, however, at the cost of added algebraic complexity. 


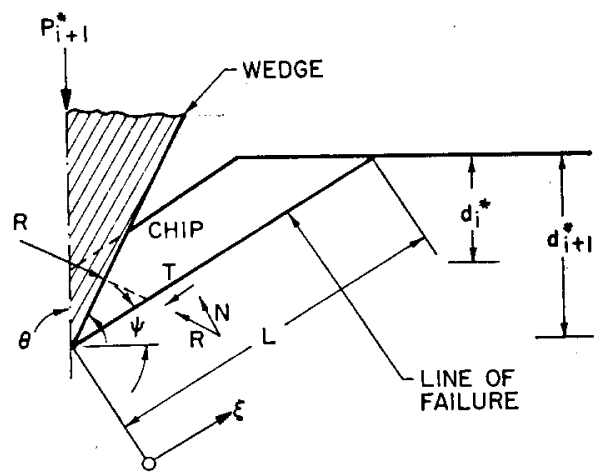

FIG. 2. Incipient chip formation.

where

$P_{i+1}^{*}=$ force per unit length of cutting edge necessary to remove the $(i+1)$ st chip $2 \theta=$ wedge angle

and $N, T$ are resultant forces per unit length of cutting edge defined by

$$
N=\int_{0}^{L} \sigma(\xi) \mathrm{d} \xi ; \quad T=\int_{0}^{L} \tau(\xi) \mathrm{d} \xi
$$

where $\sigma(\xi), \tau(\xi)$ are the normal and shear stress distributions along the failure plane, respectively. $L$ is the chip length and can be expressed:

$$
L=\frac{d_{i+1}^{*}}{\sin \psi}
$$

where $d_{i+1}^{*}$ is the penetration at the $(i+1)$ st chip removal.

The Coulomb-Mohr failure criterion [2,3], modified to account for rock anisotropy, is given by: $\dagger$

where

$$
|\bar{\tau}|-\tan \phi \sigma-\left[S_{1}-S_{2} \cos 2(\psi-\gamma)\right]=0
$$

$$
\begin{aligned}
\phi & =\text { angle of internal friction (assumed constant throughout) } \\
S_{1}, S_{2} & =\text { material constants. }
\end{aligned}
$$

The angle $(\psi-\gamma)$ measures the angle between the plane of minimum shear strength (bedding plane in this case) and the plane of failure. Note that the maximum and minimum shear strengths are $90^{\circ}$ apart and are $S_{1}+S_{2}, S_{1}-S_{2}$, respectively. The analysis further assumes that, to a first approximation, the failure criterion is satisfied everywhere along the failure plane. Equation (4) can thus be integrated over the length $L$ and a stress averaged form of the failure criterion used

$$
\text { i.e. }|\bar{\tau}|-\tan \phi \bar{\sigma}-\left[S_{1}-S_{2} \cos 2(\psi-\gamma)\right]=0
$$

where

$$
\bar{\sigma}=\frac{N}{L} ; \quad \bar{\tau}=\frac{T}{L}
$$

† Other failure criteria are available, e.g. [5], including a more detailed modification of Jaeger's criterion, i.e. [6]. It is felt, however, that the added complexities of introducing a more refined failure criterion is not justified in view of the assumptions. 
Using equations (1), (2) and (4), equation (6) can be expressed

$$
\frac{P_{i+1}^{*}}{2 d_{i+1}^{*}} \frac{\sin \psi \cos (\psi+\theta+\phi)}{\sin \theta \cos \phi}-\left[S_{1}-S_{2} \cos 2(\psi-\gamma)\right]=0 .
$$

Failure will occur at that angle $\psi$ such that the left-hand side of equation (7) has a maximum value. This is found by considering

$$
\frac{\mathrm{d}(\text { L.H.S. })}{\mathrm{d} \psi}=0
$$

with the result:

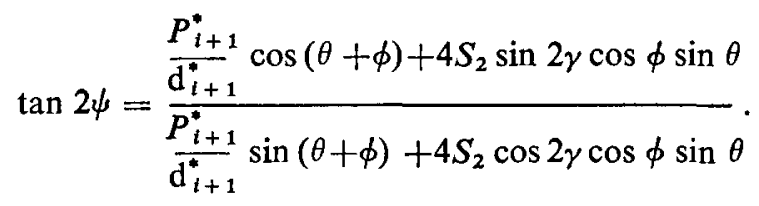

The ratio $P_{i+1}^{*} / d_{i+1}^{*}$ is still unknown, however. Substituting equation (8) into equation (7), the following quadratic equation in $P_{i+1}^{*} / d_{i+1}^{*}$ results: $\dagger$

$$
\begin{gathered}
\left(\frac{P_{i+1}^{*}}{d_{i+1}^{*}}\right)^{2} \cos ^{2}(\phi+\theta)-8 \sin \theta \cos \phi\left[S_{1} \sin (\phi+\theta)-S_{2} \sin (2 \gamma+\theta+\phi)\right]\left(\frac{P_{i+1}^{*}}{d_{i+1}^{*}}\right) \\
-16 \sin ^{2} \theta \cos ^{2} \phi\left[S_{1}^{2}-S_{2}^{2}\right]=0 .
\end{gathered}
$$

Just as in the isotropic case $P_{i+1}^{*} / d_{i+1}^{*}$ is a constant, $K$, for all $i$, dependent only on the parameters and material properties. Note that equation (9) gives the isotropic results for $S_{2}=0$ (see [1]). The positive root of equation (9) is the correct one (see Appendix A):

$$
\begin{gathered}
\frac{P_{i+1}^{*}}{d_{i+1}^{*}}=\frac{4 \sin \theta \cos \phi}{\cos ^{2}(\theta+\phi)}\left\{S_{1} \sin (\theta+\phi)-S_{2} \sin (2 \gamma+\theta+\phi)\right. \\
\left.+\sqrt{ }\left(\left[S_{1} \sin (\theta+\phi)-S_{2} \sin (2 \gamma+\theta+\phi)\right]^{2}+\left[S_{1}^{2}-S_{2}^{2}\right] \cos ^{2}(\theta+\phi)\right)\right\}=K .
\end{gathered}
$$

From equation (10) the ratio of load to penetration at chip formation is now known, however, the individual magnitudes of $P_{i+1}^{*}$ and $d_{i+1}^{*}$ are not. An additional equation is available, however, namely the crushing law:

$$
P_{\mathbf{t + 1}}=k\left[d_{t+1}-d_{t}^{*}\right]
$$

where $k$ is material constant dependent, in addition, on wedge geometry and direction of penetration. At the time of chip formation equation (11) becomes

$$
P_{i+1}^{*}=k\left[d_{i+1}^{*}-d_{i}^{*}\right] \text {. }
$$

+ See Appendix A for detailed algebra.

¥ A linear crushing law is assumed for convenience. Note that $K$, equation (10), is independent of crushing law. 
Following [1], equations (10) and (12) can be solved simultaneously to obtain $P_{i+1}^{*}$, $d_{i+1}^{*}$ in terms of $d_{i}^{*}$. Recurrence relations can then be derived which relate $P_{i+n}^{*}, d_{i+n}^{*}$ to $d_{i}^{*}:$

i.e.

$$
\begin{aligned}
& P_{i+n}^{*}=K\left(\frac{k}{k-K}\right)^{n} d_{i}^{*} \\
& d_{i+n}^{*}=\left(\frac{k}{k-K}\right)^{n} d_{i}^{*} .
\end{aligned}
$$

Thus, for $i=1$, the force and penetration levels after $n$ cycles can be related to the penetration at the first chip. Note that geometric similarity exists (due to linearity) with the similarity variable $k /(k-K)$. In Fig. 3 the actual penetration process is shown along with two idealizations (constant rate, constant load tests) which bound the actual.

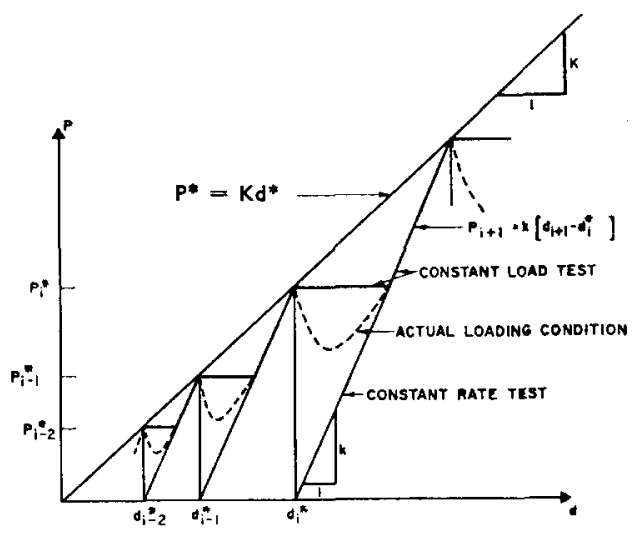

Fig. 3. Constant load and other test conditions.

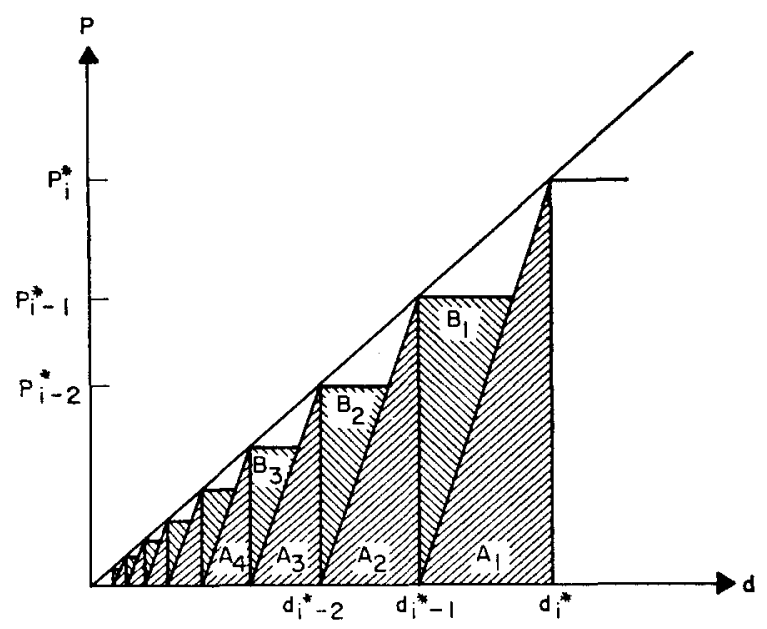

Fig. 4. Work done in constant rate test $\left(\sum_{j=1}^{\infty} A_{j}\right)$, and constant load test $\left(\sum_{j=1}^{\infty}\left(A_{j}+B_{j}\right)\right)$. 
Determination of the specific energy (energy required per unit volume of rock removed) for the constant rate, constant load tests follows the analysis of [1] exactly. It should be noted that only for the special cases included in this work $\left(\gamma=0,90^{\circ}\right)$ (symmetric cases) will the analysis be the same. The specific energies for the constant rate test and constant load test are obtained by dividing the energy consumed in each test (see Fig. 4) by the volume removed and are respectively:

$$
\begin{gathered}
E_{a}=\frac{k K}{2(2 k-K)} \tan \psi \\
E_{b}=\frac{K\left[(k-K)^{2}+k^{2}\right]}{2 k(2 k-K)} \tan \psi .
\end{gathered}
$$

In the following section numerical results are compared with some experiments on Indiana limestone.

\section{EXPERIMENTAL AND NUMERICAL RESULTS}

The pertinent experimental results [4] for Indiana limestone are listed in Table 1. For $\gamma=0^{\circ}, 90^{\circ}$ the force is perpendicular and parallel to the bedding plane, respectively, for both the compressive strength and wedge penetration tests.

The compressive strength tests were conducted on cylinders $1.75 \mathrm{in}$. in diameter and $4.75 \mathrm{in}$. long. Sample ends were finished to a tolerance of $\pm 0.005 \mathrm{in}$. and during tests were directly in contact with the platen (unlubricated).

TABLE 1. EXPERIMENTAL DATA FOR INDIANA LIMESTONE $\left(2 \theta=90^{\circ}\right)$

(Numbers in parentheses refer to number of tests making up the average)

\begin{tabular}{ccccc}
\hline$\gamma$ & $\begin{array}{c}\text { Compressive } \\
\text { strength (psi) }\end{array}$ & $\begin{array}{c}\text { Crushing } \\
\text { slope, } k \text { (psi) }\end{array}$ & $\begin{array}{c}\text { Slope of } \\
\text { envelope, } K \text { (psi) }\end{array}$ & $\begin{array}{c}\text { Specific } \\
\text { energy (psi) }\end{array}$ \\
\hline $0^{\circ}$ & $10,900^{(5)}$ & $172,900^{(6)}$ & $58,700^{(3)}$ & $3950^{(8)}$ \\
$90^{\circ}$ & $10,000^{(5)}$ & $154,900^{(4)}$ & $77,900^{(1)}$ & $7220^{(6)}$ \\
\hline
\end{tabular}

All of the wedge penetration experiments were run with a tungsten carbide wedge having a 1 in. cutting edge, a wedge angle of $2 \theta=90^{\circ}$, and a tip radius of 0.05 in. Maximum penetration depths of the order $0.15 \mathrm{in}$. were run. The rock sample was a cube approximately 12 in. on a side. Force-penetration results were taken out directly on an $x-y$ plotter and measurements of crushing slope, envelope slope, and energy were computed from the graphs. Crater volumes were obtained using a burette. The craters were first coated to prevent absorption and several readings were then averaged.

As input for the numerical results, the following items are needed: $k, 2 \theta, \phi, S_{1}$, and $S_{2}$. $k$ will be taken from the experimental results. The wedge angle is $2 \theta=90^{\circ}$. The angle of internal friction $\phi$ is difficult to establish, particularly in the vicinity of the wedge tip where the mean or hydrostatic stress can be extremely high. In any event, numerical values are computed for three different values of $\phi \dagger\left(\phi=5^{\circ}, 10^{\circ}, 20^{\circ}\right) . S_{1}$ and $S_{2}$ can be found from the following equations (see Appendix B)

$$
\begin{aligned}
& 4\left[S_{1}^{2}-S_{2}^{2}\right]+4\left[S_{1}+S_{2}\right] \mu S_{c 1}=S_{c 1}^{2} \\
& 4\left[S_{1}^{2}-S_{2}^{2}\right]+4\left[S_{1}-S_{2}\right] \mu S_{c 2}=S_{c 2}^{2}
\end{aligned}
$$

$\dagger$ Note that equations (16) cannot be used for finding $S_{1}, S_{2}$ for $\mu=0$. 
where

$S_{c 1}=$ compressive strength for $\gamma=0^{\circ}$

$S_{c 2}=$ compressive strength for $\gamma=90^{\circ}$

$\mu=\tan \phi$

Numerical values for $S_{1}, S_{2}$ as a function of $\phi$ are given in Table 2. Using equations (8), (10), (14) and (15), the chip failure angle, the slope of the envelope, and the specific energies for both cases can be computed for $\gamma=0^{\circ}, \gamma=90^{\circ}$. The results are presented in Table 3.

TABle 2. Values of THE SHEAR CONSTANTS as a FUNCTION OF $\phi$

\begin{tabular}{cccc}
\hline $\begin{array}{c}\text { Shear } \\
\text { constants } \\
\text { (psi) }\end{array}$ & $\phi=5^{\circ}$ & $\phi=10^{\circ}$ & $\phi=20^{\circ}$ \\
\hline$S_{1}$ & 5320 & 4490 & 3680 \\
$S_{2}$ & 2350 & 1090 & 460 \\
\hline
\end{tabular}

TABLe 3. Numerical COMPUTATIONS

\begin{tabular}{|c|c|c|c|c|c|c|}
\hline \multirow[b]{2}{*}{$\gamma$} & \multicolumn{3}{|c|}{ Failure angle, $\psi$} & \multicolumn{3}{|c|}{ Slope of envelope, $K$ (psi) } \\
\hline & $\phi=5^{\circ}$ & $\phi=10^{\circ}$ & $\phi=20^{\circ}$ & $\phi=5^{\circ}$ & $\phi=10^{\circ}$ & $\phi=20^{\circ}$ \\
\hline $\begin{array}{r}0^{\circ} \\
90^{\circ}\end{array}$ & $\begin{array}{l}17 \cdot 4 \\
21 \cdot 6\end{array}$ & $\begin{array}{l}16 \cdot 6 \\
18 \cdot 0\end{array}$ & $\begin{array}{l}12 \cdot 4 \\
12 \cdot 8\end{array}$ & $\begin{array}{l}40,570 \\
83,900\end{array}$ & $\begin{array}{l}55,790 \\
83,160\end{array}$ & $\begin{array}{r}92,140 \\
115,630\end{array}$ \\
\hline$y$ & \multicolumn{3}{|c|}{$\begin{array}{l}\text { Specific energy-con. rate, } E_{a} \text { (psi) } \\
\phi=5^{\circ} \quad \phi=10^{\circ} \quad \phi=20^{\circ}\end{array}$} & \multicolumn{3}{|c|}{ 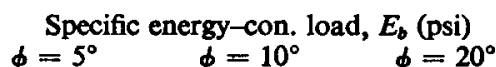 } \\
\hline $\begin{array}{r}0^{\circ} \\
90^{\circ}\end{array}$ & $\begin{array}{r}3560 \\
11,360\end{array}$ & $\begin{array}{l}4990 \\
9236\end{array}$ & $\begin{array}{r}6910 \\
10,330\end{array}$ & $\begin{array}{r}5650 \\
13,750\end{array}$ & $\begin{array}{r}7280 \\
11,130\end{array}$ & $\begin{array}{r}8420 \\
10,995\end{array}$ \\
\hline
\end{tabular}

These results are discussed and compared with the experimental data in the following section.

\section{DISCUSSION OF RESULTS}

The experimental results for Indiana limestone illustrated in Table 1, indicate a rather interesting behavior; namely, a much larger difference in specific energy in perpendicular directions than in the corresponding material properties. An extension of an existing theory has been outlined herein which predicts at least qualitatively the same trends (see Table 3). Several problems exist. While the trends are established, a large amount of scatter was evident in the experimental results. The numbers presented represent a simple average of available data. Concerning the theory, it should be pointed out that not all input data (material properties) is clearly established, e.g. the angle of internal friction $\phi$. Two major drawbacks of the theory are the assumptions made in the analysis and the inability of the theory to adequately describe the initial phases of the penetration process. These questions are discussed in more detail by PAUL and SIKARSKIE [1]. In spite of this, however, the theory 
is in good agreement qualitatively, i.e. it provides an explanation of the difference in specific energies in the two directions. It is also felt that quantitative agreement is reasonable in view of the statistical nature of the material, and the simplifying assumptions of the analysis.

From Table 3 it is evident that the numerical results are very sensitive to the angle of internal friction $\phi$. Comparing Tables 1 and 3 for $\phi=10^{\circ}$ indicates that the computed envelope slope is in good agreement with the experimental value. This is somewhat inconclusive, however, due to the limited test data. Concerning the specific energies, although the same trends exist in the numerical data for $\gamma=0^{\circ}, 90^{\circ}$, the numerical results are uniformly higher than the experimental. For $\phi=10^{\circ}$, the experimental value of the specific energy lies outside the 'bounds'. It is felt that this is due to the assumption of the failure criterion being satisfied along the entire failure plane.

Several interesting features appear in the analysis. As anisotropy increases, i.e. as $S_{c 1}-$ $S_{c 2}$ increases, it can be shown that the differences in the failure angle $\psi$ and the specific energies for $\gamma=0^{\circ}, \gamma=90^{\circ}$ also increases, as is expected. It is also interesting to note that the slope of the envelope is larger in the direction parallel to the bedding plane. This indicates the possibility of wedge forces larger for penetrations parallel to the bedding than perpendicular (for equal penetration distances). This is opposite to the crushing phase where the crushing slope is largest perpendicular to the bedding plane. The physical difference in specific energies is also related to this question, i.e. since $K$ is larger, $k$ is smaller for $\gamma=90^{\circ}$, more energy is consumed in the penetration. Also, the failure angle is larger for $\gamma=90^{\circ}$ leading to smaller volumes. Both of these effects lead to larger specific energies for $\gamma=90^{\circ}$.

Acknowledgment-The authors would like to express their sincere appreciation to the Department of the Interior Bureau of Mines for the support of this work under Grant No. MIN-10.

\section{REFERENCES}

1. Paul B. and Sikarskie D. L. Preliminary theory of static penetration by a rigid wedge into a brittle material. Trans. AIME 232, 372-383 (1965).

2. JAEGER J. C. Shear failure of anisotropic rocks. Geol. Mag. 96, 55-72 (1960).

3. JAEGER J. C. Elasticity, Fracture and Flow, 2nd edition, Wiley N.Y. (1962).

4. Sikarskie D. L. Experiments on Wedge Indentation in Rocks, Ingersoll-Rand T. N.-262. Ingersoll-Rand Research Center, Princeton, New Jersey (May 1966).

5. WAlsh J. B. and BRACE W. F. Fracture criterion for brittle anisotropic rock. J. geophys. Res. 69, (16) 3449-3456 (1964).

6. MCLamore R. and Gray K. E. The mechanical behavior of anisotropic sedimentary rocks. J. basic Engng Trans. ASME 88, 62-76 (1967).

\section{APPENDIX A}

$$
\text { Derivation of the Quadratic Equation in } \frac{P_{i+1}^{*}}{d_{i+1}^{*}}
$$

The failure criterion is given by equation (7) with the failure angle $\psi$ defined by equation ( 8 ). If we further define

$$
K_{1}=\frac{P_{i+1}^{*}}{2 d_{i+1}^{*}} \frac{1}{\sin \theta \cos \phi}
$$

the failure criterion can be written in the following form

$$
K_{1} \sin \psi \cos (\phi+\theta+\psi)-\left[S_{1}-S_{2} \cos 2(\psi-\gamma)\right]=0
$$


expanding:

$$
\begin{aligned}
& K_{1}[\sin 2 \psi \cos (\theta+\phi)-\sin (\theta+\phi)(1-\cos 2 \psi)] \\
& -2\left[S_{1}-S_{2}(\cos 2 \gamma \cos 2 \psi+\sin 2 \gamma \sin 2 \psi)\right]=0 .
\end{aligned}
$$

Dividing by $\cos _{m}^{2} 2 \psi$ and collecting terms;

$$
\begin{aligned}
& {\left[K_{1} \cos (\theta+\phi)+2 S_{2} \sin 2 \gamma\right] \tan 2 \psi} \\
& \quad+\left[K_{1} \sin (\theta+\phi)+2 S_{2} \cos 2 \gamma\right] \\
& \quad-\left[2 S_{1}+K_{1} \sin (\theta+\phi)\right] \sqrt{ }\left(1+\tan ^{2} 2 \psi\right)=0 .
\end{aligned}
$$

This has the form

$$
A \tan 2 \psi+B-C \sqrt{ }\left(1+\tan ^{2} 2 \psi\right)=0
$$

where $A, B$, and $C$ can be determined from equation (A.4). Note also, from equation (8), that:

$$
\tan 2 \psi=\frac{A}{B} .
$$

Substituting (A.6) into (A.5) we find:

$$
A^{2}+B^{2}-C \sqrt{ }\left(A^{2}+B^{2}\right)=0
$$

ort

$$
A^{2}+B^{2}=C^{2} .
$$

Substituting back for $A, B, C$, and $K_{1}$, and rearranging, the final form results.

$$
\begin{gathered}
\left(\frac{F_{i+1}^{*}}{d_{i+1}^{*}}\right)^{2} \cos ^{2}(\theta+\phi)-8 \sin \theta \cos \phi\left[S_{1} \sin (\theta+\phi)-S_{2} \sin (2 \gamma+\theta+\phi)\right]\left(\frac{P_{i+1}^{*}}{d_{i+1}^{*}}\right) \\
-16 \sin ^{2} \theta \cos ^{2} \phi\left[S_{1}^{2}-S_{2}^{2}\right]=0 .
\end{gathered}
$$

This quadratic equation has a positive and a negative root. The negative root means the slope $\frac{P_{i+1}^{*}}{d_{i+1}^{*}}$ is negative which"has no physical meaning.

\section{APPENDIX B}

\section{Computation of the Shear Constants $S_{1}, S_{2}$}

$S_{1}$ and $S_{2}$ are not directly known but can be obtained in terms of the compressive strengths in the two directions. This is done by reducing the biaxial failure criterion to two independent uniaxial cases. The biaxial failure criterion given by JAEGER [2] is:

$$
\mu^{2}\left(C_{m}+a\right)^{2}-\left(1+\mu^{2}\right)\left(\tau_{m}+b\right)^{2}=S_{2}^{2}-b^{2}\left(1+\mu^{2}\right)
$$

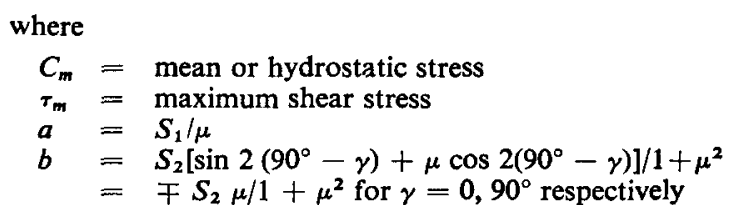

Two special cases of equation (B.1) are now considered, namely uniaxial compression perpendicular and parallel to the bedding plane.

First case, $\gamma=0$

$$
C_{m}=\tau_{m}=S_{c 1} / 2
$$

† The set of roots introduced by squaring, i.e. $A^{2}+B^{2}=0$, are imaginary. 
Substituting into equation (B.1)

$$
\mu^{2}\left(\frac{S_{c 1}}{2}+\frac{S_{1}}{\mu}\right)^{2}-\left(1+\mu^{2}\right)\left(\frac{S_{c 1}}{2}+\frac{S_{2 \mu}}{1+\mu^{2}}\right)^{2}=S_{2}^{2}-\frac{S_{2}^{2} \mu^{2}}{1+\mu^{2}}
$$

simplifying:

$$
4\left(S_{1}^{2}-S_{2}^{2}\right)+4\left(S_{1}+S_{2}\right) \mu S_{c 1}=S_{c 1}^{2} .
$$

Second case, $\gamma=90^{\circ}$

$$
C_{m}=\tau_{m}=S_{c 2} / 2 .
$$

Substituting into equation (B.1) and simplifying:

$$
4\left(S_{1}^{2}-S_{2}^{2}\right)+4\left(S_{1}-S_{2}\right) \mu S_{c 2}=S_{c 2}^{2} .
$$

Equations (B.2) and (B.3) are identical to equations (16). 